\title{
Visual Decision-Making in Real-Time Business Intelligence: A Social Media Marketing Example
}

\author{
Elena Davcheva \\ Technical University Darmstadt \\ davcheva@ise.tu-darmstadt.de
}

\author{
Alexander Benlian \\ Technical University Darmstadt \\ benlian@ise.tu-darmstadt.de
}

\begin{abstract}
This paper presents a study into the use of visualizations in real-time business intelligence. Different visualization designs for a social media marketing use case are tested and evaluated through the lens of cognitive load theory. By reducing the complexity of visualizations and subsequently cognitive load, end-users can achieve markedly improved decision-making performance in situations where time is critical and data is fast-paced.
\end{abstract}

\section{Introduction}

In the digital age consumer engagement is becoming more dynamic, while companies attempt to make marketing relevant to the needs of the individual consumer, and available to them in the moment they engage with a particular need [32]. Approaches like moment marketing and micro-moments must beat consumer "tune-out" and be quick enough [1] to deal with decreasing time spent per browsing session [1], as well as more users consulting Internet sources in various stages of the consumer journey - when looking at product options, or even in the store right before making a purchase [37]. In this new environment, real-time business intelligence (RTBI) is an indispensable support for companies in capturing and delivering market and customer insights in real time and enabling an immediate response.

RTBI has already become indispensable when working with social media. For example, airlines use social media as a tool for customer service and impression management - American Airlines on average responds to customer tweets within 10 minutes [18]. Furthermore, real-time ad placements and social media monitoring are used to improve customer relationships and increase revenue [33]. RTBI applied to social media opens the opportunity for companies to capitalize on current events as they unfold and engage with consumer product demand as it is generated.
According to Chen et al., traditional BI can be defined as a complex system of "technologies, systems, practices, methodologies, and applications that analyze critical business data to help an enterprise better understand its business and market and make timely business decisions" [8]. RTBI expands the traditional BI pipeline by using real-time data [7] as a way to deal with the terabytes of data that companies deal with on a daily basis [30]. Applying RTBI successfully in organizations is challenged by the need to accommodate aspects of human decision-making RTBI users are expected to make decisions under conditions of cognitive and time constraints at any given moment [38]. Such tools incorporate data visualization as a proven means to present data to endusers in an efficient and effective manner [25]. Prior research in the field points out that visual interfaces for RTBI solutions must be designed with different user needs in mind than traditional BI [12]. Namely, RTBI visualizations must support decision-making on the fly using a constantly updated view, which creates a high cognitive load for the analyst [12]. Thus, fundamentally a suitable RTBI visualization must consolidate higher cognitive demands from constantly incoming data with the need for quick decisionmaking.

Thus far there is sparse research addressing the design and data requirements when visualizing RTBI data. In their overview of the state of RTBI research, Nadj and Schieder [27] identified the following research gap: "How shall the user interface of RTBI systems be designed to support the decision maker's cognitive abilities and thereby regulate the degree of information consumption?" The authors' review points that the cognitive constraints of decision- makers have thus far not been properly examined within the context of RTBI [27]. This may cause suboptimal decisionmaking performance and results in critical and timesensitive situations [28].

This paper addresses the above research gap by examining user performance on RTBI visualizations applied to social media (as a frequent industry use case for RTBI), through the lens of cognitive load. We 
conduct an experiment to test the influence of varying amounts of visual information on real-time decisionmaking. We show that leaner visual solutions are superior to more complex interfaces in high-pressure situations where data is in motion and decisions need to be made on the spot. Owing to a lower cognitive load, simpler data visualizations lead to higher certainty and accuracy, as well as faster decisionmaking. We further provide a basis for future RTBI data visualization design.

The subject of this study does not cover long-term BI analytics where business insights must be derived based on trend development and data characteristics over time; instead, we focus on the social media use case where business context changes swiftly and requires taking immediate decisions [11].

The remainder of this paper is organized as follows. First, a theoretical background of cognitive load is presented, followed by hypothesis development; after, we explain our methodological approach, followed by a presentation of experiment results; finally, we discuss our results and draw conclusions.

\section{Theoretical background}

First introduced in the late 1980's, cognitive load theory is concerned with optimizing the absorption of new information by using the correct presentation format for a given purpose [35]. Three types of cognitive load can be distinguished: extraneous (the format in which information is presented), intrinsic (type of task to be completed based on the given information), and germane (resources to acquire longterm knowledge) [35]. By minimizing extraneous load, a proper presentation format will allow more cognitive resources for an individual to cope with intrinsic and germane load, thus leaving more room to understand the information presented, to reason, and draw conclusions or plan actions [29]. According to the theory, when a user observes a visualization, visual cues are first processed by sensory memory, then only the most relevant information is forwarded to working memory; therefore, in a highly cluttered visualization not all visual cues will eventually reach working memory [19]. A visualization with too many cues presents a challenge for working memory, and potentially leads to information overload and poor performance. The more elements and change working memory has to process, the higher the chances that something will not be noticed or will be quickly forgotten [35].

Cognitive load theory has been used previously in several data visualization studies as means to capture how users process visual stimuli. For example, people working with graphs have difficulty tracing graph patterns such as relationships and other interactions once the graph becomes overly complex and cognitive load increases as a result [19]. Another example is a visual internet monitoring system which improves the reaction time and accuracy of end-users by automatically reducing the amount of visual data displayed [39].

High cognitive load has been shown to lead to negative decision-making performance in a variety of fields such as economics, medicine, and even personal interactions. In medicine, medical professionals make worsened decisions under higher cognitive load and tend to revert to social prejudices when deciding on patient treatments, thus hurting minority patients [6]. In economics, higher cognitive load leads to deteriorated decisions in various situations [10]. Allen et. al. conducted an experiment using visualizations of uncertainty data under high cognitive load. Respondents' ability to grasp basic characteristics of the visualized data did not deteriorate, however their ability to deliberately process and make decisions was negatively affected by the higher cognitive load, resulting in suboptimal choices [2]. Higher cognitive load is also shown to negatively affect judgment of other individuals. Gilbert et. al. showed that higher cognitive load leads to a lower use of situational cues when assessing others' personalities, thus leading to preconceived biases and social prejudice [16].

The evidence from prior literature overwhelmingly shows that higher cognitive load will negatively affect visual reasoning and decision-making in various scenarios. Thus far, RTBI research has not considered the cognitive limitations of decision-makers, nor have significant strides been made in the direction of optimizing the user interface, which affects many critical functions of RTBI such as minimizing decision latency or human error [27]. We address the research gap by conducting an experiment based on prior research on the interplay between human cognition and visual perception. For example, Huang et. al. note that visualizations become more useful when they go beyond simply allowing the user to understand the data displayed, and actually "support human reasoning"; understanding and reasoning visual information is conditioned upon adequate cognitive resources, and a reduction of cognitive load resulting from a visualization directly enables this [19].

In light of these findings in prior research, and in combination with the conditions of real-time decisionmaking, we hypothesize that a lower cognitive load from visual BI data will accommodate the needs for quick and effective reasoning, which arise in a realtime decision-making environment. 


\section{Hypotheses}

Based on the theoretical background and the literature review, we construct hypotheses to test in an experiment. The hypotheses of the study test for the relationship between cognitive load, change perception, and decision-making. In terms of decisionmaking, we look at decision accuracy, decision certainty, and time taken to decide. Change perception is measured by how well respondents perceive change in direction and variability.

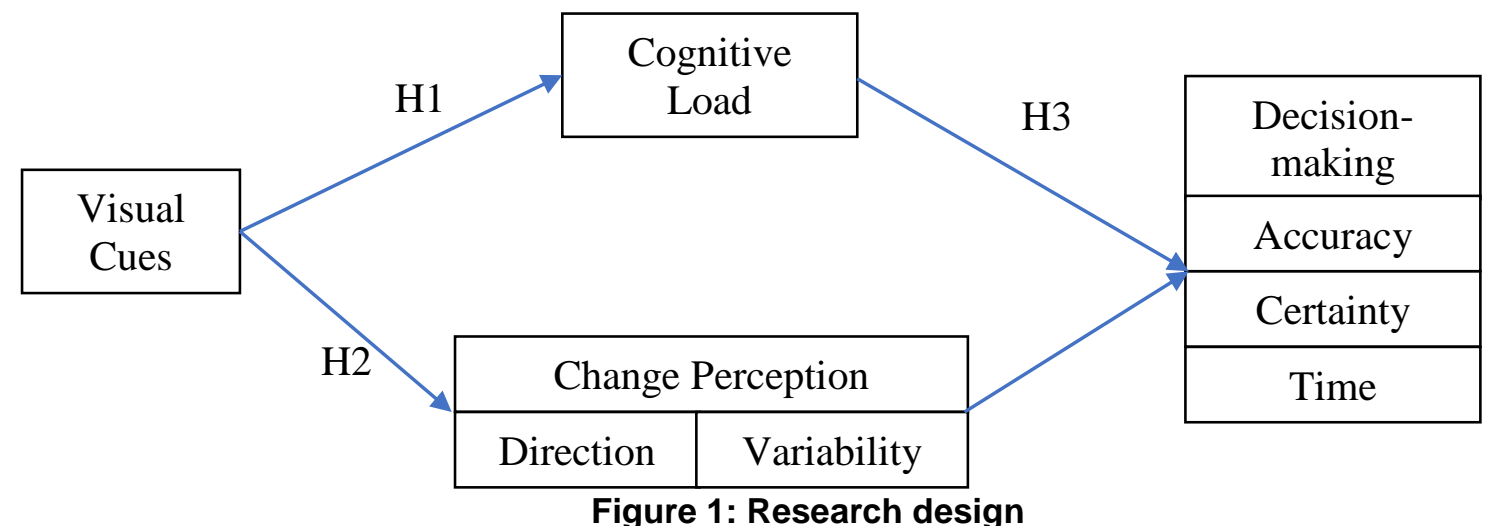

To begin with, we test for different cognitive load levels for complex and simple visualizations. The goal of the complex visualization is not to purposefully confound and overwhelm users, but to provide more information than the simple visualization in order to measure whether the additional visual cues help or detract from the goal of taking real-time decisions based on real-time information. The complexity of business data and the requirements of real-time analysis and decision-making need to be reconciled with the amount of cognitive load a user can cope with and still achieve reasonable performance in such an environment. According to the definition of cognitive load theory, extraneous load is higher in visualizations with more visual cues [29]; therefore, we hypothesize that in RTBI it will translate to higher mental effort that does not enhance decision- making.

H1: Compared with simple visual cues, complex visual cues will increase cognitive load at the expense of realtime decision-making performance.

Furthermore, we hypothesize that users' ability to perceive change as a major factor in dynamic real-time visualizations will also affect the quality of their decisions [17]. Namely, since visualizations that introduce higher cognitive load hinder change perception [36], this will lead to poor performance. By examining the correlation between self-reported cognitive load and change perception, we can look into how this affects decision-making. In measuring change perception, we look at aspects important for decisionmaking: noticing variability (stable and predictable vs. erratic change) and changes in direction of movement (increase vs. decrease) [2].

H2a: Compared with simple visual cues, complex visual cues will negatively affect direction of change perception in real-time decision making.

H2b: Compared with simple visual cues, complex visual cues will negatively affect visual data variability perception in real-time decision making.

Finally, respondents' certainty in their choices will serve as a check if they are confident in their decisions or rather estimating. Self-assessment of certainty will also show if visual complexity affects decision certainty and whether there is a relation between certainty and decision accuracy, as well as time [23]. This is a crucial aspect of real-time decision making, where hesitation might cost time, which in turn translates to potential loss of value [20] [21].

H3a: Compared with simple visual cues, complex visual cues will decrease decision certainty in realtime decision-making.

H3b: Compared with simple visual cues, complex visual cues will decrease decision accuracy in realtime decision making.

H3c: Compared with simple visual cues, complex visual cues will increase decision time in real-time decision making. 


\section{Experimental setup and measures}

In order to test our hypotheses, we conduct an experiment with visualization versions as a betweensubject factor. A survey was set-up with a scenario where the respondent is a business analyst required to place a product advertisement on a social media platform in real time. The scenario uses data from the social media site Reddit (reddit.com). Reddit is an appropriate platform for such an example, as topics of conversations dwindle quite rapidly, but can produce significant buzz in a short time [3].

In the survey scenario, respondents were required to choose a page to advertise the Microsoft X-Box gaming console. In order to decide, survey participants used a set of 4 visualizations - a simple and a complex version of a scatterplot and a bubble graph, respectively. The scatterplot illustrates in which subpages of Reddit people talked about the X-Box. The bubble graph visualizes the words used in conversations about the X-Box. After each version of the scatterplot, respondents chose on which page they would advertise the X-Box; furthermore, they selfreported cognitive load, indicated how certain they are in their choice, as well as how well they perceived changes in the data.

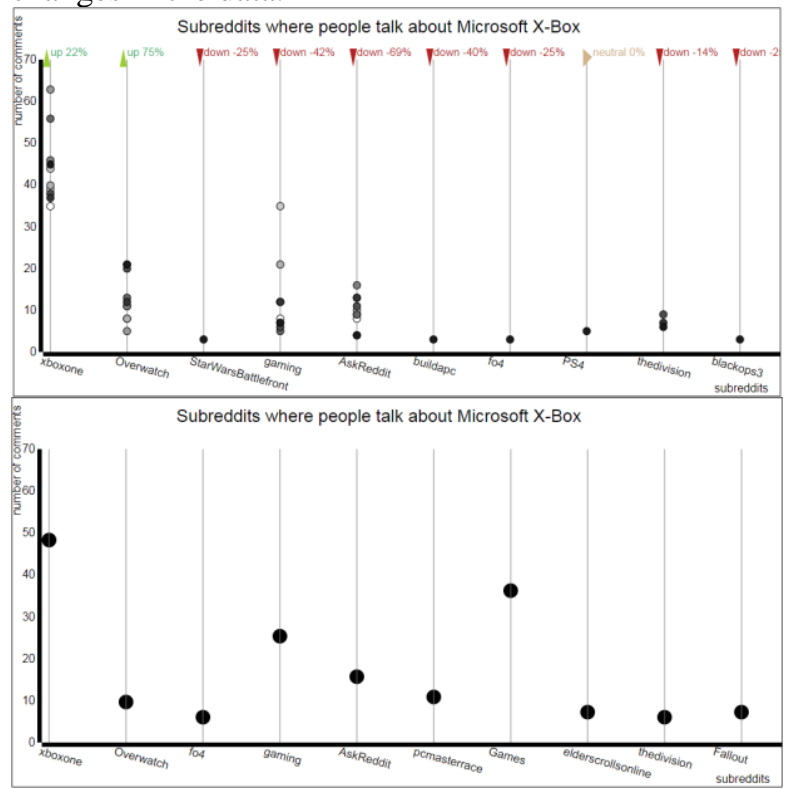

Figure 2. Complex and simple scatterplot (trailing past values as well as percentage change cues are missing in the simple version)

Two different visualization designs were used to ensure that the effects of cognitive load on decisionmaking can be generalized across different visualization designs. Scatterplots plot the relationship between two variables on a vertical and horizontal axis and can compare the state of several categories in parallel, as well as illustrate the trail (path of change) of a variable [14]. Scatterplots were selected for this study in order to capitalize on the ability to visualize past variable values as a way to add visual complexity [31]. Other visualizations customarily used with categorical data such as parallel coordinates would not be able to visualize a variable's past values without adding visual density through overlapping lines [26]. For word visualization, bubble charts provide size and color as cues to display frequency change [31]. Word clouds, which are traditionally used to visualize word frequency, are meant for static representations [9].

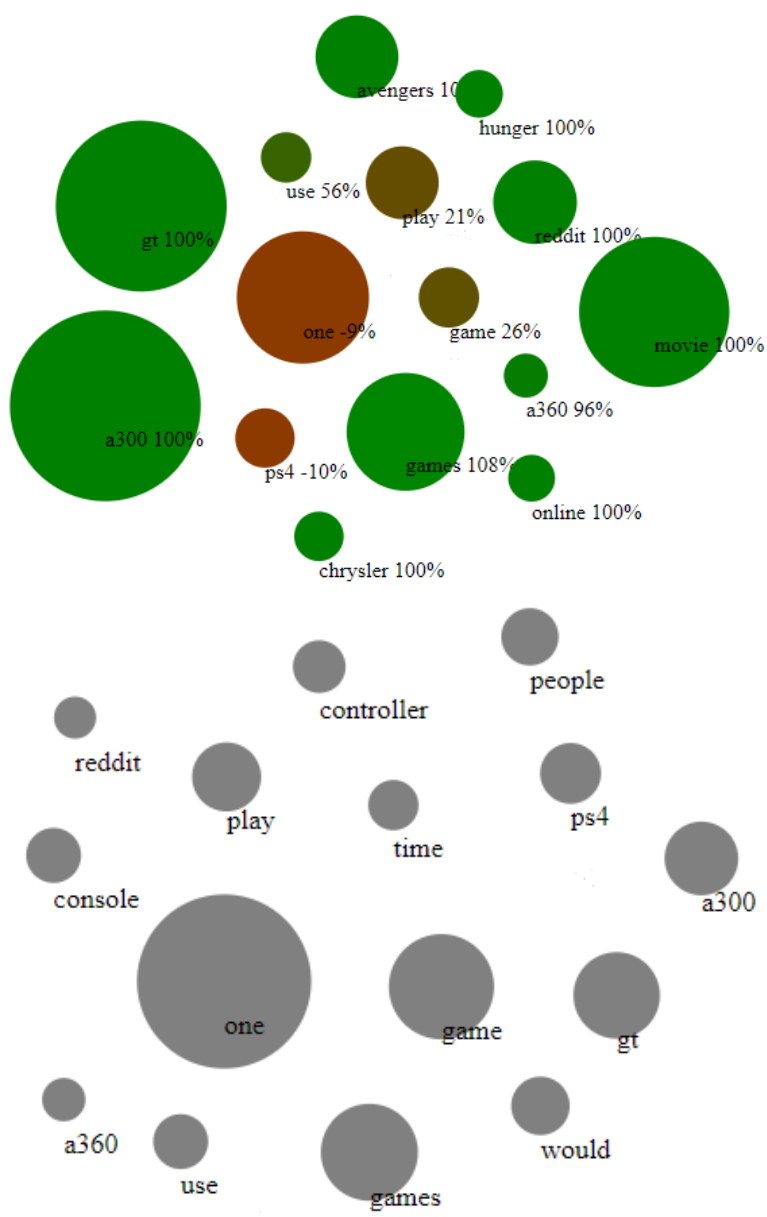

Figure 3. Complex and simple bubble graph (color as direction change indication as well as percentage change cues are missing in the simple version)

The visualizations are programmed with the D3 library [5]. The data displayed is dynamically updated every 10 seconds - the interval was selected after preliminary tests showed that shorter update intervals 
are too fast for most participants. The visualizations display 2 minutes' worth of Reddit data - any comments across the entire website mentioning the $\mathrm{X}$ Box gaming console would be visualized. All survey participants view visualizations of the same underlying dataset.

The following table summarizes the measurement items to be tested in hypotheses. Borrowing from previous studies which have employed the measures used in this experiment, all measures except time and accuracy are measured on a 7-point Likert scale. We follow the approach of Paas \& van Merriënboer to measure cognitive load [29],

] as it has been shown to be reliable and sufficient in previous visualization studies [24]. In order to measure decision accuracy, we follow Kobsa's analysis approach [22]. Finally, the traditional information systems metrics for user perceptions of usefulness (PU), ease of use (PEOU), and satisfaction [ag] are included as a complementary perspective to the hypotheses as to how user perception compares to user performance in this case [13].

Table 1. Measures used

\begin{tabular}{ll}
\hline \multicolumn{1}{c}{ Measure } & Source \\
\hline Cognitive load & {$[29]$} \\
Decision accuracy & {$[22]$} \\
Decision certainty & {$[21]$} \\
Decision time & {$[20]$} \\
\hline
\end{tabular}

Change perception measures

User perception of direction change [21]

User perception of variability

[2]

User preference measures

Perceived usefulness

Perceived ease of use

Satisfaction
Table 2. Visualization sets

\begin{tabular}{lll}
\hline & Scatterplot & Bubble graph \\
\hline $\begin{array}{l}\text { Simple } \\
\text { Version (no } \\
\text { added cues) }\end{array}$ & $\begin{array}{l}\text { No trail, no } \\
\text { percentage change } \\
\text { indicator, no } \\
\text { direction of } \\
\text { change indicator }\end{array}$ & $\begin{array}{l}\text { No color change, } \\
\text { no percentage } \\
\text { change indicator }\end{array}$ \\
& $\begin{array}{l}\text { Trail, percentage } \\
\text { change indicator, } \\
\text { Complex }\end{array}$ & $\begin{array}{l}\text { Color indicating } \\
\text { direction of } \\
\text { Version }\end{array}$ \\
(added & $\begin{array}{l}\text { color indicating } \\
\text { direction of } \\
\text { cues) }\end{array}$ & $\begin{array}{l}\text { percentage change } \\
\text { indicator }\end{array}$ \\
\hline
\end{tabular}

Each of the two visualizations has two versions: one with and one without complex cues, summarized in the table below. The experiment implements a complex version as means to increase cognitive load and see if additional information will improve or deteriorate user decision behavior.

\section{Results}

The experiment was conducted online using the micro-task website Amazon Mechanical Turk. After accounting for manipulation checks, there were 72 completed responses for each of the four visualization versions. Respondents' answers are tested using ANOVA, as well as $\chi 2$ for choice accuracy. Order effects were offset by using counterbalancing. For the most part, no significant differences were found among respondents based on sex, age, occupation, gaming experience, and most importantly experience working with data visualizations. One exception is that scatterplot users with more gaming experience are significantly more accurate in their choices $(p<0.01)$. Additionally, bubble graph users with more gaming experience recorded better perception of change direction $(\mathrm{p}=0.044)$.

To begin with, respondents for both visualizations reported that cognitive load was higher when using the complex visualizations $(\mathrm{p}<0.001)$. Thus, the theoretical assumption of cognitive load theory and $\mathrm{H} 1$ is confirmed. 
Table 3. Analysis results

\begin{tabular}{rllll}
\hline Variable & Presentation format & Mean square & F (df) & Sig. \\
\hline Cognitive Load & Scatterplot & 18.78 & $6.74(1)$ & 0.010 \\
& Bubble graph & 24.17 & $8.51(1)$ & 0.004 \\
\hline Decision Certainty & Scatterplot & 21.78 & $12.28(1)$ & 0.001 \\
& Bubble graph & 14.06 & $7.13(1)$ & 0.008 \\
\hline Decision Time & Scatterplot & 8755500 & $64.75(1)$ & 0.000 \\
& Bubble graph & 2245626 & $20.56(1)$ & 0.000 \\
\hline PU & Scatterplot & 0.06 & $0.032(1)$ & 0.859 \\
& Bubble graph & 1.43 & $0.58(1)$ & 0.447 \\
\hline PEOU & Scatterplot & 4.00 & $1.75(1)$ & 0.188 \\
& Bubble graph & 6.67 & $2.64(1)$ & 0.014 \\
\hline Satisfaction & Scatterplot & 1.17 & $0.50(1)$ & 0.489 \\
& Bubble graph & 4.70 & $1.51(1)$ & 0.041 \\
\hline Direction of change & Scatterplot & 0.007 & $0.02(1)$ & 0.894 \\
& Bubble graph & 2.51 & $4.14(1)$ & 0.045 \\
\hline Variability & Scatterplot & 0.44 & $0.68(1)$ & 0.412 \\
& Bubble graph & 1.56 & $2.15(1)$ & 0.145 \\
\hline
\end{tabular}

Regarding choice accuracy, we followed the approach of Kobsa (2001) and implemented a $\chi 2$-test to compare the accuracy across different levels of cognitive load for both visualizations [22]. The optimal choice of page for ad placement was made according to the data, i.e. pages are ranked according to the number of conversations revolving around the X-Box. Summary data of choices is presented in the graphs below.

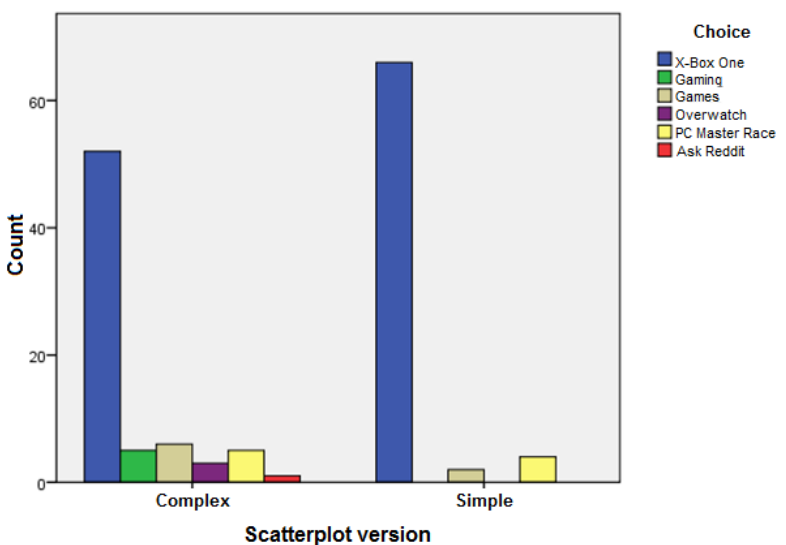

Figure 4. Scatterplot choice accuracy (X-Box One is the optimal choice)

Across both visualizations there is a significant difference in the accuracy of answers given between the two versions $(p<0.001)$. For the scatterplot, the optimal possible choice (based on the number of conversations revolving around the $\mathrm{X}$-Box console) is the page of "X-Box One", and this was chosen by 62 and 71 of all 72 respondents for both versions of the scatterplot. Not only the final choice, but also the variety of responses selected plays a role - in general, for both visualizations, the complex version leads to more variety in selected pages and words for ad placement.

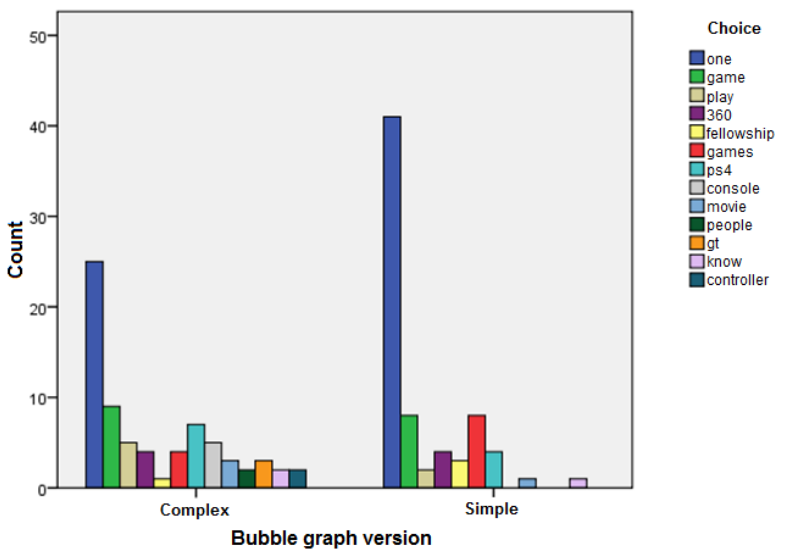

Figure 5. Bubble graph choice accuracy (the word "one" is the optimal choice)

However, responses based on the complex version introduced 3 more options and therefore a lower accuracy altogether. This suggests that the additional information displayed on the complex scatterplot (past pages with X-Box-related conversations, as well as the precise percentage change as opposed to only visual change) led to respondents considering more options. The same can be observed for the bubble graph, in that choice of words is much more concentrated (57\% vs. $35 \%$ for the complex and simple bubble graph respectively chose the most frequently shown word "One"). Thus, H3b is confirmed. 
Table 4. Mean decision times in seconds

\begin{tabular}{|l|l|l|}
\hline & Scatterplot & Bubble graph \\
\hline Simple & $875 \mathrm{~s}$. & $613 \mathrm{~s}$. \\
\hline Complex & $375 \mathrm{~s}$. & $350 \mathrm{~s}$. \\
\hline
\end{tabular}

Test results confirmed hypothesis H3a. Respondents reported feeling significantly more certain in their choice of page when using the simple scatterplot $(\mathrm{p}<0.001)$ as well as the simple bubble graph $(\mathrm{p}>0.05)$.

Both visualizations confirm hypothesis $\mathrm{H} 3 \mathrm{c}$ in that the average time for both simple visualizations was about $50 \%$ lower than that for complex ones $(\mathrm{p}<0.001)$ In order to avoid order effects, the experiment implemented counterbalancing in that the four versions of the visualizations came in a randomized order to each respondent. Further analysis showed that the order in which visualizations were displayed does not influence the time taken to answer.

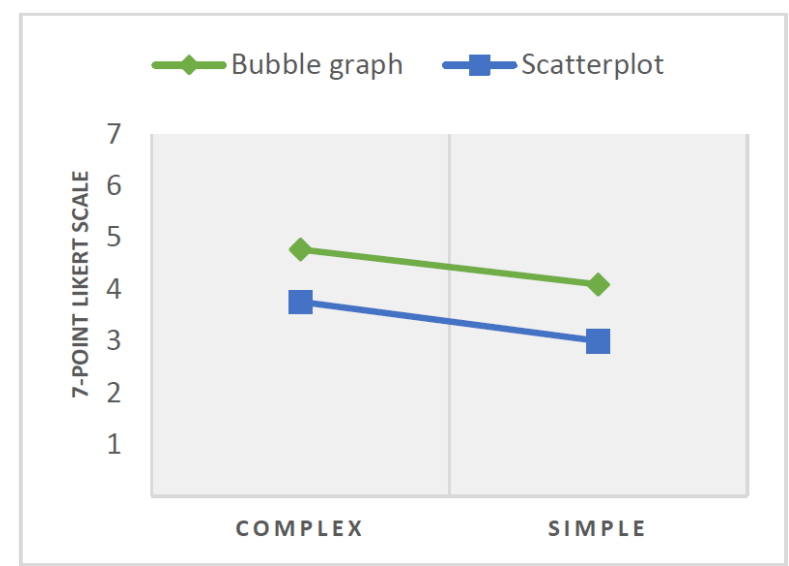

Figure 6. Estimated marginal means for cognitive load

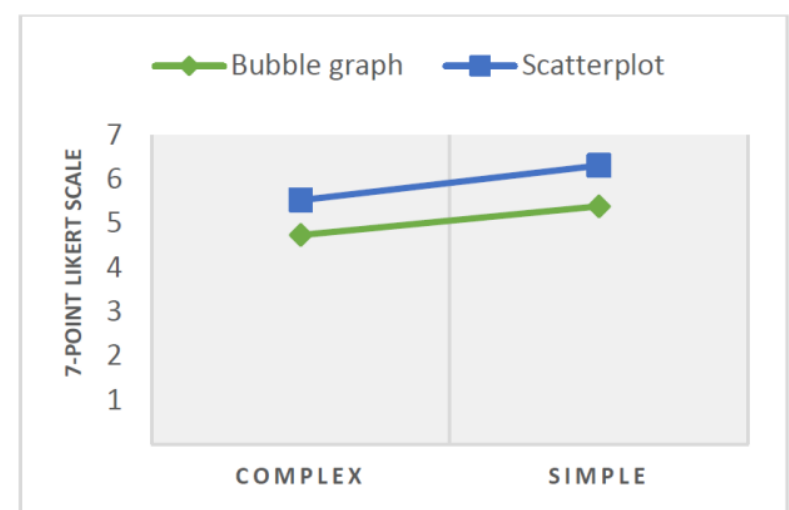

Figure 7. Estimated marginal means for certainty

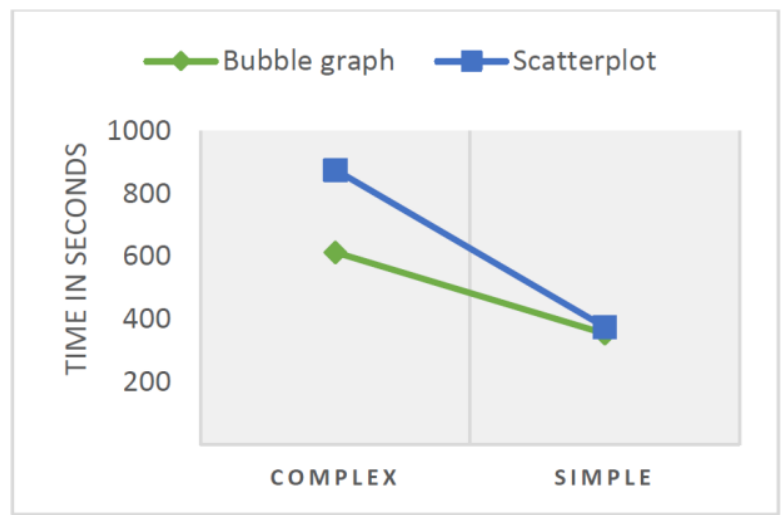

Figure 8. Estimated marginal means for decision time

We fail to confirm hypothesis $\mathrm{H} 2 \mathrm{~b}$ regarding variability for both visualizations ( $p$-values 0.412 and 0.145 for the scatterplot and bubble graph respectively). There is a significantly improved perception of direction of change for the complex bubble graph. This may be due to the lack of axes, which renders the use of color as a signal of direction change (red being decrease and green being increase) a significant aid in this area. The results suggest that a simpler visualization does not add to a better change perception, however at the same time this does not negatively influence decision making. Furthermore, the improved change perception for the complex bubble graph did not lead to better decision-making metrics.

Prior studies such as the one conducted by Toker et. al. have shown that there is at least a partial connection between visual perception and user preferences, however findings have been inconclusive [34]. We present the findings from our study to complement performance and perception metrics for a comprehensive view on user decision-making behavior. For the scatterplot, respondents do not find a significant difference between versions in terms of PU $(\mathrm{p}=0.859)$, PEOU $(\mathrm{p}=0.188)$, and satisfaction $(\mathrm{p}=$ 0.489 ), however for the bubble graph users find that the simpler version was both easier to use, as well as more satisfactory $(\mathrm{p}<0.05)$. Users were also asked why they prefer one version over the other, where for the simple bubble graph they pointed that it was "less visually cluttered", followed by "it hides unnecessary details". Interestingly, whereas the update speed for all visualizations was the same, respondents perceived the speed as more adequate on the simple version, meaning that more cues lead to things seemingly moving faster.

\section{Discussion}

This study uses a social media use case to show that simple visualizations have decisive decision-making 
advantages in a real-time BI context. Reducing the visual cues displayed on a visualization reduces cognitive load while simultaneously improving decision accuracy and slashing time needed to decide, as well as increasing user certainty. We show that change perception is not much improved in either complex or simple visualization versions, with the exception of the complex bubble graph. However, the improved change perception for the complex bubble graph did not lead to better decision-making metrics.

In terms of the bubble graph, preference and performance are positively correlated, however even when preferences are not significantly stronger, as for the scatterplot, performance is still better with a simpler visualization. This implies that personal user preferences are not a reliable indicator of whether or not a visualization will render improved decisionmaking support.

Since the results of all decision-making hypotheses are the same for both scatterplot and bubble graph, the results are largely generalizable to various visual designs.

\section{Implications and future work}

\subsection{Theoretical implications}

The study contributes to the already existing body of work in data visualizations and expands it to the area of RTBI based on cognitive load theory while applying a novel viewpoint: empowering RTBI decision-makers by simplifying visualizations by way of reducing cognitive load, which leads to reduced decision times, improved accuracy and increased decision certainty.

We make an addition to RTBI visual metrics by combining change perception - an established metric in visual perception literature -and showing that it does not strongly affect decision-making performance. Finally, we add to the existing studies that have explored the relationship between visual preference and performance by showing that user preferences are not a stable performance indicator when it comes to RTBI visualizations.

\subsection{Practical implications}

This study bears important practical implications for architects of RTBI systems. As data visualization becomes an increasingly important analytics tool, and real-time intelligence becomes more widely adopted by businesses, professionals in the field will have to find ways to design effective visuals for real-time business situations. We show that RTBI visuals benefit from a lean design rather than crowded interfaces.

Additionally, our study bears specific design implications for visualizations that lack axes to indicate a positive or negative direction of change. By applying a green-to-red gradient in a bubble graph, user perception of direction change improves significantly.

\subsection{Limitations and future work}

Real time business intelligence and specifically RTBI visualizations is an area where IS researchers have yet to make their mark. First, our study does not address how much is "enough" in terms of visual cues that would give adequate and sufficient information to a decision-maker under time pressure. Second, more research needs to be done regarding axes, scale, and value change orientation that users need for different visualization designs.

Furthermore, user preferences appear to be an unreliable signal for the appropriateness of visualizations regarding decision-making. Further investigation is possible in this direction by testing whether user-driven preference adjustments in realtime aid or hurt decision-making performance.

Finally, the study results are based on Amazon Mechanical Turk participants, who may not be representative for broader populations. Future research should include samples from diverse sources.

\section{Conclusion}

In the digital era, companies who work with the user who is "always on" must deal with limited attention spans [15], and a short-lived use of social media platforms [4]. In this fast-paced environment, real-time intelligence will enable marketers and analysts to capitalize on short-term trends and conversations. As different types of organizations grapple with real-time data more frequently in their daily business, the question of optimizing visual analytics is becoming more relevant. This paper attempts to inform the design of visualizations for realtime decision-making by investigating the relation between visual complexity, human cognition, and the nature of human decision-making. Essentially, our study shows that more visual cues will only serve to prolong and confuse the decision-making process, while at the same time only marginally improving change perception, which however does not positively influence decision-making capabilities. By simplifying their visuals, visual analysts can optimize the real-time decision-making process. 


\section{References}

[1] Adams, L., Burkholder, E., and K. Hamilton, "MicroMoments: Your Guide to Winning the Shift to Mobile", Google, 2015. Retrieved from www.thinkwithgoogle.com

[2] Allen, P. M., Edwards, J. A., Snyder, F. J., Makinson, K. A., and D. M. Hamby, "The effect of cognitive load on decision making with graphically displayed uncertainty information”, Risk Analysis, 34(8), pp. 1495-1505, 2014.

[3] Anderson, K.E., "Ask me anything: what is Reddit?" Library Hi Tech News, 32(5), pp. 8-11, 2015.

[4] Asur, S., Huberman, B.A., Szabo, G. and C. Wang, "Trends in social media: Persistence and decay", Proceedings of the 5th ICWSM, Palo Alto: AAAI Press, p. 434, 2011.

[5] Bostock, M., "Data-Driven Documents", Program documentation, D3.js, N.p., n.d. Retrieved from https://d3js.org/

[6] Burgess, D. J., “Are providers more likely to contribute to healthcare disparities under high levels of cognitive load? How features of the healthcare setting may lead to biases in medical decision making", Medical Decision Making, 30(2), pp. 246-257, 2010.

[7] Chaudhuri, S., Dayal, U. and V. Narasayya, "An overview of business intelligence technology", Communications of the ACM, 54(8), pp.88-98, 2011.

[8] Chen, H., Chiang, R.H.L., and V.C. Storey, "Business Intelligence and Anlaytics: From Big Data to Big Impact", MIS Quarterly, 36(4), pp. 1165-1188, 2012.

[9] Cui, W., Wu, Y., Liu, S., Wei, F., Zhou, M. X., and Qu, H., "Context preserving dynamic word cloud visualization", Visualization Symposium (PacificVis), IEEE Pacific, pp. 121-128, 2010

[10] Deck, C. and S. Jahedi, "The effect of cognitive load on economic decision making: A survey and new experiments", European Economic Review, 78, pp. 97-119, 2015.

[11] Fisher, T., "ROI in social media: A look at the arguments", Journal of Database Marketing \& Customer Strategy Management, 16(3), pp. 189-195, 2009.

[12] Fischer, F., and D. A. Keim, "NStreamAware: Realtime visual analytics for data streams to enhance situational awareness", Proceedings of the Eleventh Workshop on Visualization for Cyber Security, pp. 65-72, ACM, 2014.

[13] Franz, M., Scholz, M., and O. Hinz, "2D versus 3D Visualizations in Decision Support - The Impact of Decision Makers' Perceptions", International Conference on Information Systems (ICIS), 2015.
[14] Friendly, M. and D. Denis, "The early origins and development of the scatterplot", Journal of the History of the Behavioral Sciences, 41(2), pp. 103-130, 2005.

[15] Gausby, A., “Attention Spans”, Consumer Insights, Microsoft Canada, 2015.

[16] Gilbert, D. T., and R. E. Osborne, "Thinking backward: Some curable and incurable consequences of cognitive busyness", Journal of Personality and Social Psychology, 57(6), p. 940, 1989.

[17] Goldsberry, K., and S. Battersby, "Issues of change detection in animated choropleth maps", Cartographica: The International Journal for Geographic Information and Geovisualization, 44(3), pp. 201-215. 2009.

[18] Gunarathne, P., Rui, H., and A. Seidmann, "Customer Service on Social Media: The Effect of Customer Popularity and Sentiment on Airline Response", 48th Hawaii International Conference on Systems Science (HICSS), pp. 3288-3297, IEEE, 2015.

[19] Huang, W., Eades, P., and S. H. Hong, "Measuring effectiveness of graph visualizations: A cognitive load perspective", Information Visualization, 8(3), pp. 139-152, 2009.

[20] Iyilikci, O., Becker, C., Güntürkün, O., and S. Amado, "Visual processing asymmetries in change detection", Perception, 39(6), pp. 761-769, 2010.

[21] Kiani, R., Corthell, L., and M. N. Shadlen, "Choice certainty is informed by both evidence and decision time", Neuron, 84(6), pp. 1329-1342, 2014.

[22] Kobsa, A. "An empirical comparison of three commercial information visualization systems", INFOVIS IEEE Symposium on Information Visualization, pp. 123130, IEEE, 2001.

[23] Kunimoto, C., Miller, J. and H. Pashler, "Confidence and accuracy of near-threshold discrimination responses", Consciousness and cognition, 10(3), pp. 294-340, 2001.

[24] Leutner, D., Leopold, C., and E. Sumfleth, "Cognitive load and science text comprehension: Effects of drawing and mentally imagining text content", Computers in Human Behavior, 25(2), pp. 284-289, 2009.

[25] Liu, S., Cui, W., Wu, Y. and M. Liu, "A survey on information visualization: recent advances and challenges", The Visual Computer, 30 (12), pp. 1373-1393, 2014.

[26] McDonnell, K. T. and K. Mueller. „Illustrative parallel coordinates", Computer Graphics Forum, 27(3), pp. 10311038), 2008.

[27] Nadj, M., and C. Schieder, "Quo Vadis Real-Time Business Intelligence? A Descriptive Literature Review and 
Future Directions", European Conference on Information Systems (ECIS), 2016.

[28] Nadj, M., Morana, S. and A. Maedche, "Towards a situation-awareness-driven design of operational business intelligence and analytics systems", Proceedings of the 10th International Conference on Design Science Research in Information Systems and Technology (DESRIST). pp. 3340, 2015.

[29] Paas, F., Tuovinen, J. E., Tabbers, H., and P. W. Van Gerven, "Cognitive load measurement as a means to advance cognitive load theory", Educational psychologist, 38(1), pp. 63-71, 2003.

[30] Pareek, A. (2010). Addressing BI Transactional Flows in the Real-Time Enterprise Using GoldenGate TDM. In M. Castellanos, U. Dayal, \& R. J. Miller, eds. BIRTE 2009. pp. 118-141.

[31] Robertson, G., Fernandez, R., Fisher, D., Lee, B. and J. Stasko, "Effectiveness of animation in trend visualization", IEEE Transactions on Visualization and Computer Graphics, 14(6), pp. 1325-1332, 2008.

[32] Steimer, S., "Digital Next Practices: Micro-moment Marketing", American Marketing Association, 2016. Retrieved from

https://www.ama.org/publications/MarketingNews/Pages/di gital-marketing-micromoments.aspx

[33] Stone, M. D, and N. D. Woodcock, "Interactive, direct and digital mareting: A future that depends on better use of business intelligence", Journal of Research in Interactive Marketing, 8(1), pp. 4-17, 2014.
[34] Toker, D., Conati, C., Carenini, G., and M. Haraty, "Towards Adaptive Information Visualization: On the Influence of User Characteristics", Proceedings of the 19th International Conference on User Modeling, Adaptation, and Personalization (UMAP), pp. 274-285, 2012.

[35] Van Merrienboer, J. J. and J. Sweller, "Cognitive load theory and complex learning: Recent developments and future directions", Educational psychology review, 17(2), pp. 147-177, 2005.

[36] Vergauwe, E., Langerock, N., and P. Barrouillet, "Maintaining information in visual working memory: Memory for bindings and memory for features are equally disrupted by increased attentional demands", Canadian Journal of Experimental Psychology, 68(3), p. 158. 2014.

[37] Vinerean, S., Cetina, I., Dumitrescu, L. and M. Tichindelean, "The effects of social media marketing on online consumer behavior", International Journal of Business and Management, 8(14), p.66, 2013.

[38] Visinescu, L. L., Jones, M. C., and A. Sidorova, "Improving Decision Quality: The Role of Business Intelligence", Journal of Computer Information Systems, 57(1), pp. 58-66, 2017.

[39] Yelizarov, A. and D. Gamayunov, "Adaptive Visualization Interface That Manages User's Cognitive Load Based on Interaction Characteristics", Proceedings of the 7th International Symposium on Visual Information Communication and Interaction, ACM, 2014. 